\title{
A Model to Assist Pedagogical Scenario Building Process in cMOOCs
}

\author{
Aicha Bakki ${ }^{1,2}$, Lahcen Oubahssi ${ }^{1}$, Sébastien George ${ }^{1}$, Chihab Cherkaoui ${ }^{2}$ \\ ${ }^{1}$ UBL, University of Maine, EA4023, LIUM \\ 72085, Le Mans, France \\ \{aicha.bakki, lahcen.oubahssi, sebastien.george\}@univ-lemans.fr \\ ${ }^{2}$ Ibn Zohr University, IRF-SIC Laboratory \\ 80000, Agadir, Morocco \\ c.cherkaoui@uiz.ac.ma
}

\begin{abstract}
This paper focuses on pedagogical scenario building process in cMOOCs. We aim to offer solutions to support teachers in the design, deployment and adaptation of such courses. To achieve this goal, we propose a generic cMOOC oriented pedagogical scenario building process. In adequacy with cMOOC aspects, this process allows to describe learning environments' elements, roles and pedagogical activities in this massive, open and connectivist situation. Moreover, the proposed model is generic in the sense that it allows to create scenarios regardless of a particular context or domain.
\end{abstract}

Keywords- MOOC; cMOOC; pedagogical scenario; pedagogical scenario building.

\section{INTRODUCTION}

Since 2008, MOOC (Massive Open Online Courses) have become prominent, democratizing learning and online education. With their advent, MOOCs have attracted a large number of learners around the world, regardless of their education, gender or age. These courses have different characteristics such as: massiveness, openness to all and accessibility on a large scale, certifications' nature, content nature, evaluation methods, etc. Some research has addressed specific issues related to one or more of these characteristics. We can mention in particular works related to MOOCs' taxonomies [1], massive traces' analysis [2], learner profiles [3], adaptation through various criteria, namely: learning styles, learners' preferences, etc. [4], modalities of updating MOOC scenario and scenario aspects [5] etc.

This work focuses mainly in the last two aspects. The first concerns pedagogical scenarios design, especially scenario building process for a connectivist MOOC (cMOOC) and its deployment. Pedagogical scenario building aims to formalize the course design process. In our work, this process must take into account massiveness of these environments, their openness and connectivist characteristics. The second aspect concerns adaptation and personalization issue of designed scenarios. This component is fundamental to respect the cMOOCs specificities. Specifically, this aspect is related to the generation of personalized scenarios for a particular learner or group of learners, according to learners' needs, learning objectives, profiles and preferences.

The main idea of this paper is to present some results about scenario building process for a cMOOC and its deployment. We first introduce an overview of research related to cMOOCs, from a pedagogical scenario perspective. We focus on theoretical approach of connectivism in order to demonstrate the importance and the need of a scenario building process in such environments. Then we detail our proposal through a cMOOC oriented scenario model. Finally, we present tools that we had developed to assist scenario design, its production and its deployment; through a workflow-oriented approach via the use of BPMN standard.

\section{RESEARCH CONTEXT AND AIMSH}

cMOOCs are based on the connectivist approach which promotes learning through collaboration, production, sharing and creation of peer-to-peer connections. Connections emerge when individuals share the same goals; connected communities are formed and produce resources [6]. These courses are based on four principles: the aggregation of information and resources, a reflection on these resources that can be linked to new knowledge (remixing), the creation of new resources (production) and finally the sharing of newly produced resources [6].

The first principle of connectivism developed by Siemens stipulates that learning and knowledge are associated to the diversity of opinions and resources [6]. In order that this diversity shows results, it seems important to provide an organized learning process. The ability to evaluate the relevance of information and opinions is also emphasized as an indispensable skill to deploy, in order to deal with the overabundance of resources. Pedagogical scenario building is therefore a process of modeling the learning situation. It's about designing content, organizing resources, planning activities and mediating to induce and conduct learning [7]. A scenario implies notions of roles, resources, activities and orchestration. It aims to organize and structure the learner's activity, to define the role of each actor and the relationship with the used tools and resources.

Overall, these environments create new challenges for involved actors, namely teacher and learner. For teachers, it is to really think about learning by formalizing, scripting and implementing courses using appropriate technologies and procedures. Therefore, it is necessary to create favorable conditions and contexts for teachers to design their courses. Our hypothesis resides in the proposal of a 
pedagogical scenario process adapted to various connectivist aspects. For learners, many advantages can be advanced, such as: flexibility, accessibility, openness, collaboration, autonomy, etc. The openness involves, among other things, the freedom of learners to choose their learning objectives and define which activities are most suitable for them. In this context, creation of new learning resources is important: learners play the role of teachers and have to create and produce new resources and knowledge. In this sense, we consider that learners must be guided and oriented during this process in order to develop and promote their autonomy and offering them the possibility to define pedagogical scenarios for themselves and their collaborative groups.

Although many researchers support the idea of the need for a scenario building process in cMOOCs [5] [8], there is not yet, to our knowledge, research that focuses specifically on the technical and methodological aspects. In our view, the question is not only to produce a model that meets connectivist specifications, but also to define an adaptable model, both by teachers and learners. The aim of this proposal is to offer to teachers a methodological guide to design cMOOC, and to give learners the flexibility to define their objectives, and also to adapt pedagogical scenarios dynamically.

\section{CMOOC-ORIENTEd PEDAGOGICAL SCENARIO LIFE CYCLE}

To meet the requirements described above, we propose a cMOOC-oriented cycle in three phases: (1) Formalization. The aim of this phase is to provide teachers with tools and / or models to assist them to design formalized cMOOCoriented scenario. There are several pedagogical scenariobuilding models. The most important is IMS Learning Design model [8]. According to [9], the IMS-LD model lacks expressiveness regarding description of users' interactions during collaborative tasks. This limitation does not allow describing activities in which learners are in strong interaction, as with cMOOCs. (2) Deployment. The aim of this phase is to operationalize pedagogical scenario in a cMOOC platform. Various works dealt with pedagogical scenarios operationalization, like [10] by example. Our contribution consists in proposing an operationalization process by offering to teachers' services to design their pedagogical scenarios independently of any platform. In that way, we chose BPMN standard (Business Process Model and Notation) [11] in formalization phase. This standard will generate pedagogical scenarios from which we can extract modular XML files to be transformed and imported into the targeted platform (Open edX, Coursera, ...). (3) Adaptation. This phase is designed in respect of one of the connectivism aspects through our proposal: learner is an actor of his/her learning; s/he is not anymore a consumer of resources but a creator. Each learner is dynamically involved in the continuing process of pedagogical scenario building. As mentioned above, the pedagogical scenario building process, in connectivist environments, is immersing throughout the learning session. Thus, our objective is to bring a solution to the emergence of needs throughout the learning process by giving to learners a marge of expressivity allowing them, under the control of the teachers, to propose new scenarios, for them and their peers.

\section{From CMOOC Pedagogical SCENARIO TO THE LEARNING PLATFORM}

\section{A. General View of the Proposed Model}

The proposed model allows expressing specific structural properties of connectivist environment as well as their temporal properties. The model is composed of three levels: (1) The cMOOC oriented pedagogical scenario model, that describes pedagogical scenarios related to connectivism principles. It defines and then associates pedagogical concepts needed for the design of an abstract scenario. This model will be embedded in our editor, which allows teachers creating scenarios. We also aim to allow learners to personalize scenarios according to their needs, learning objectives, profiles and preferences. (2) The abstract pedagogical scenario, which is an instance of the cMOOC oriented pedagogical scenario model. This scenario describes activities that take place in a cMOOC environment, their sequences, the resources needed for their realizations, the actors who realize them, the type of interaction, etc. (3) The operationalized pedagogical scenario, which represents instantiation of the pedagogical scenario (2) on a particular platform.

\section{B. cMOOC Oriented Pedagogical Scenario Model}

In this section, we present the elements of the proposed model. As shown in Figure 1, the model covers the different entities. First, objectives' have to be defined, with two levels of description. The first level describes the achievement objectives: these are the activities that the learner must be able to perform. The second level concerns the learning objectives targeted by each learner. A MOOC is organized on weeks; each week contains lessons; each of these lessons has one or more pedagogical objectives. Pedagogical activities are described in "Activity part," they represent: learning activities to be undertaken by the learners or the evaluation activities. The categorization of these activities is related to connectivist aspects. Thus, an activity can be assigned to one of five categories: aggregating (regrouping consulting and cognition activities), remixing (including communication, sharing and metacognition activities), repurposing (including production and collaboration activities), feed forwarding (sharing activities) and evaluating (referring to evaluation activities). We distinguish, also the activities sequencing and the type and nature of the activity in a connectivist environment. For its operationalization, the "Activity part" needs: "Environment Part" which contains resources and tools needed to carry out activities and produce resources. This entity describes environment characteristics. It describes pedagogical resources (provided and produced one), their links with 
activities, and used tools (Forum, Wiki, social networks, etc.). Finally, "User part," defines different roles to be played in pedagogical scenarios and type of possible interactions. Each actor who plays a role must perform activities associated with that role. Moreover, within the connectivist framework, collaborative activities are important. It is therefore necessary to define interaction type.

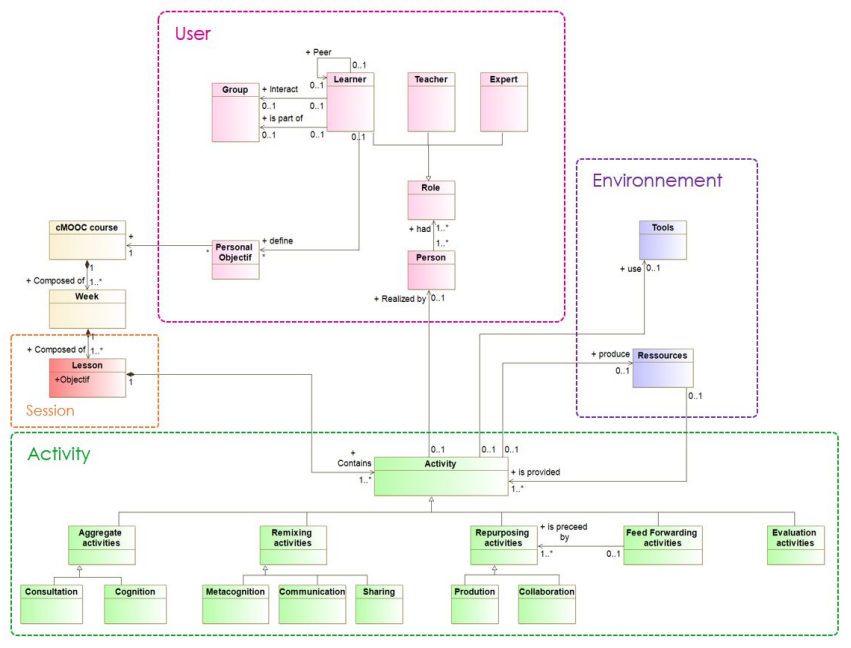

Figure 1. cMOOC-oriented scenario model

\section{Tools \& Applications}

In order to deploy the cMOOC-oriented scenario model, we have developed two tools (Figure 2). The first one is a pedagogical scenario editor. This editor offers functionalities to create and modify different components of a scenario including, learning environments, users and activities. The editor provides teachers with simple features for the scenario visualization and manipulation, by hiding the underlying conceptual concepts. It offers a range of tools designed specifically to facilitate cMOOC-oriented scenarios design by respecting organizational concepts of these environments. We remind that our editor is based on BPMN standard. It provides a graphical interface to design pedagogical scenarios by keeping the design process as simple as possible [11]. In this sense, the use of the BPMN standard seems promising. Our proposal accurate to our conceptual framework, by proposing a pedagogical workflow which is centered on connectivist activity.

The second tool we have developed is an API to deploy and execute generated scenarios. Thus, we provided a solution to transform the workflow designed through our editor into an operationalized scenario.

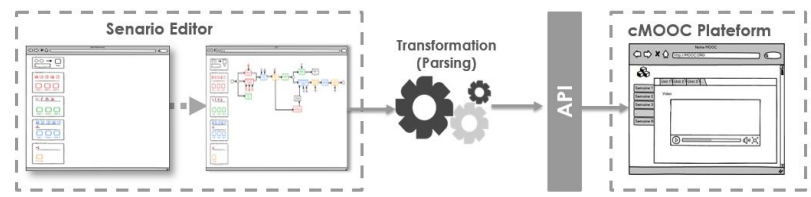

Figure 2. Pedagogical scenario's deployment

\section{CONCLUSION}

In this paper, we presented an approach based on modeling for pedagogical scenario building of cMOOC activities. We proposed models and tools allowing scenario creation and exploitation. The starting point for developing our scenario model was to identify information needed to describe a cMOOC scenario. To identify this information, we relied on the concepts used in the connectivist approach, enriched through case studies of existing cMOOC. The scenario should also describe the type of interaction, since collaborative work is a very important concept in cMOOCs. Finally, it should take into consideration learners' control over their learning, and their personal objectives.

We propose to teachers a tool, which allows a visual scenario design without prior knowledge of the language nor the model. Our work continues, in order to validate the current prototype with different users (teachers, designers). We had carried out a first validation of our proposal in order to demonstrate its expressivity by executing an existing cMOOC example. Finally, we plan to finalize our cMOOC oriented scenario cycle by implementing the adaptation phase. We aim to offer a dynamic adaptation by proposing minimal coverage of existing profiles and offering varied adapted scenarios to learners.

\section{REFERENCES}

[1] O. Pilli and W. Admiraal, "A Taxonomy of Massive Open Online Courses," Contemp. Educ. Technol., vol. 7, no. 3, pp. 223-240, 2016.

[2] G. Siemens et al., "Open Learning Analytics: An Integrated and Modularized Platform: SOLAR,” 2011.

[3] P. Hill, Some validation of MOOC student patterns graphic. 2013. Avalaible: http://mfeldstein.com/validation-mooc-student-patternsgraphic

[4] B. Hmedna, A. E. Mezouary, and O. Baz, "An Approach for the Identification and Tracking of Learning Styles in MOOCs," in Europe and MENA Cooperation Advances in Information and Communication Technologies, Springer, Cham, 2017, pp. 125-134.

[5] R. Bachelet, "La scénarisation pédagogique des MOOCs, " Rencontres nationales sur la e-formation, 2014.

[6] G. Siemens, "Connectivism: A learning theory for the digital age," In International Journal of Instructional Technology and Distance Learning, 2005.

[7] R. Koper and C. Tattersall, "Learning Design: A Handbook on Modelling and Delivering Networked Education and Training, " Springer, Heidelberg, 2005.

[8] R. Koper, "Modeling units of study from a pedagogical perspective : the pedagogical meta-model behind EML," 2001.

[9] D. Hernández-Leo et al., "COLLAGE: A collaborative Learning Design editor based on patterns," J. Educ. Technol. Soc., vol. 9, no. 1, pp. 58-71, 2006.

[10] Z. Tadjine, L. Oubahssi, C. Piau-Toffolon, and S. Iksa, "Rethinking Learning Design for Learning Technologies: A Formalized Vision to Operationalize Pattern-Based Scenarios," in 2016 IEEE 16th International Conference on Advanced Learning Technologies (ICALT), 2016, pp. 57-61.

[11] J. Da Costa, "BPMN 2.0 pour la modélisation et l'implémentation de dispositifs pédagogiques orientés processus," University of Geneva, 2014 